\title{
Placeholder prescriptions undoing years of progress in fight against antibiotic resistance
}

The British Dental Association (BDA) has warned that ongoing difficulties in accessing dental services risk fuelling the parallel health crisis of antibiotic resistance, as new data from the UK Health Security Agency underline the surge in prescriptions in the dental sector.

While all other healthcare settings recorded further drops in antibiotic prescribing, the English surveillance programme for antimicrobial utilisation and resistance (ESPAUR) Report ${ }^{1}$ shows dentistry alone saw an increase of almost a fifth in 2020 . However, patient volumes fell by more than half that year. COVID restrictions have radically reduced patient numbers, and staff were advised to adopt an 'AAA' model during the first lockdown - advice, analgesics, or antibiotics.

Antibiotic prescribing in the NHS dental settings had been steadily decreasing from 2016 to 2019 ( 0.16 and 0.13 items per 1,000 inhabitants per day respectively, $-18.4 \%$ ).

This decline was interrupted in 2020 with an increase of $17.6 \%$ reported (from 0.13 to 0.15 items per 1,000 inhabitants per day).

Data show primary care general practice accounts for $84.2 \%$ of prescribing for items per 1,000 inhabitants per day. This was followed by dentistry (10.3\%).

Dentist leaders have stressed that the government must do more to increase access sustainably and ensure patients - particularly urgent cases - have the time for operative interventions, to ensure they are not offered antibiotics as a placeholder. The current target-based NHS contract in operation in England has long represented a key barrier to progress.

A failure to address the problem of antibiotic resistance could result in ten million deaths, ${ }^{2}$ every year, globally by 2050 . As the pandemic continues and into the future, the BDA has stressed it is essential that dentists are supported to once again reduce the rate of antibiotics prescribed.
BDA Chair Eddie Crouch said:

'Antimicrobial resistance poses an even greater threat to human health than COVID. Sadly, the pandemic has wiped out years of progress in bringing down antibiotic use in dentistry.

'Placeholder prescriptions are now filling deficits in access and time, which the government has chosen not to factor in. Ministers have a responsibility to help dentists turn the page.'

\section{References}

1. UK Health Security Agency. English surveillance programme for antimicrobial utilisation and resistance (ESPAUR) report. 10 October 2014, updated 17 November 2021. Available at: https://www.gov. uk/government/publications/english-surveillanceprogramme-antimicrobial-utilisation-and-resistanceespaur-report (accessed November 2021).

2. House of Commons, Health and Social Care Committee. Antimicrobial resistance. Eleventh Report of Session 2017-19. 2018. Available at: https:// publications.parliament.uk/pa/cm201719/cmselect/ cmhealth/962/962.pdf (accessed November 2021). 